\title{
The Flora of Montreal Island.
}

\author{
By Robert Campbell, D.D., M.A.
}

For some jear's I have felt, in common with others interested in our local Natural History, that it is a pity we have not a complete list of the plants growing on our own island, and when giving a paper in March, 1891, on the Summer Wild Flowers of Great Britain, I volunteered to do what I could porsonally to repair the want, an undertaking which the Natural History Society was pleased to approve. Throughout the season of 1891, I kept my eyes open where ever I chanced to be on the island, in the prosecution of duty or in pursuit of recreation. and I succeeded in securing specimens of the following plants, which I have mounted and presented to the museum of the Natural History Society. After I had entered on my voluntary task, the Botanical Club of Canada was formed, during the sitting of the Royal Society in this city in 1891, and it has mapped out the Dominion for Botanical purposes, assigning each county or group of counties to certain well known naturalists who are asked to take the oversight of the botanical research in their respective districts, securing the co-operation of local worker's in this department, cheering them on and directing their enthusiasm. Anything I can do in connection with this joint movement, I shall be glad to attempt, and if the results I reach can be utilized by the Botanical Club, I put them entirely at its disposal. On the other hand, as one person cannot be expected to fall upon everything that grows on the island, I crave the help of every botanist in the district in the effort to secure as complete a catalogue as possible of our local flora. A well assorted cabinet of the plants growing around the city, placed in the museum of the Natural History Society, and thus put within reach of all among our citizens that are interested in this delightful science, would be a great boon; and the wonder is that an attempt to secure it has not been long ago made. 
I am not overlooking the Holmes' collection, nor am I unmindfinl of its great scientific value. But it should not be forgotten that seventy years have elapsed since it was made, and very great and important changes must have come over the floral of the district in the interval. Besides, Dr. Holmes' Herbarium did not profess to be solely a Montreal island collection, much less did it claim to present a complete catalogue of the plant-life of the island. The bulk of the specimens which he preserved for the instruction of later generations, as might be expected, were indeed gathered in the neighborhood of the city, picked up, many of them, doubtless, while he was prosecuting his professional work. To this extent, it may be taken as a complete Montreal collection, that it represerts the island plants which came under Dr. Holmes' notice, or of which he became possessed. Other places are credited occasionally as the habitat of the specimens embraced, but whenever a plant was found in or near the city, Montreal was invariably first mentioned among the localities in which it grew. When Montreal is not credited with a plant, it may therefore be taken for granted that $D_{r}$. Holmes never came across the plant on the island. It will be interesting to note how far the flora of Montreal to-day corresponds with that of seventy years ago; and I have compared the collection I have so far made with the Herbarium of Dr. Holmes, with the object of finding out what changee, if any, have come about. Or rather I have taken the catalogue of the Herbarium, prepared by the late Prof. James Barnston, and published in "The Canadian Naturalist and Geologist," for" April, 1859, as the basis of comparison; and I shall assume that Prof. Barnston's nomenclature is correct and in agreement with the classification of Gray, as it claimed to be.

Clematis Virginiana, L.-Common Virgin's Bower.August. Mountain Park, south of Park Ranger's house. (Holmes).

Clematis verticillaris, DC. - Virgin's Bower-Mountain above Ravenscrag and elsewhere. (Holmes' Atragene Americana). June. 
Anemone Virginiana, Gray. - Virginian Anemone.North end of mountain, and common on the island. June.

Anemone Pennsylvanica, L.-Pennsylvanian Anemone.(Holmes). June. Common.

Hepatica acutiloba, DC.--Sharplobed Hepatica.--North end of Mount Royal. April and May.

Hepatica triloba, Chaix._Round-lobed Hepatica.-_South end of Mount Royal, and Petite Cote Woods. (Holmes)April and May.

Thalictrum dioicum, L.-Early Meadow-rue.-Common. (Holmes). April and May.

Thalictrum polygamum, Mubl., (T. Cornuti, Holmes).-_Fall Meadow-rue.-Common. July and August.

Ranunculus abortivus, L.-Smail-flowered Crowfoot:Everywhere. (Holmes). April and May.

Ranunculus sceleratus, L._Cursed Crowfoot.-Wet meadow at Hochelaga. June.

Ranunculus repens, L.-Creeping Crowfoot. - Common. July and August. (Holmes).

Ranunculus acris, L.-Tall Crowfoot or Buttercup.June, September. Common. (Holmes).

Ranunuculus fascicularis. - Early Crowfoot.-Hochelaga bank. May.

Ranunculus septentrionalis, Poir.-Northern Crowfoot.Prince Arthur street. May.

Caltha palustris, L.-Marsh Marigold.-May. Meadows, St. Cunegonde and Lachine. (Holmes).

Coptis trifolia, Salisb.-Three-leaved Gold Thread.-May, Mount Royal and Hochelaga banks. (Holmes).

Aquilegia Canarensis, L._-Wild Columbine.-West side Mt. Royal, common. May and June. (Holmes).

Aquilegia vulgaris._Common Garden Columbine.-Escaped from cultivation, St. Laurent road. May and June.

Actcea spicata, L., var. rubra. Michx._-Red Baneberry.Common. May. (Holmes). 
Actéa alba, Bigel.-White Baneberry.-Common. May. (Holmes).

Caulophyllum thalictroides, Michx.-Blue Cohosh.-North end of mountain and elsewhere. May.

Nuphar advena, Ait._Common Yellow Pond Lily.--River St. Pierre. July. (Holmes).

Chelidonium majus, L.-Celındine.-Fletcher's Field, common. (Holmes). May.

Sanguinaria Canadensis.-Bloodroot.-Common. April. (Holmes).

Dicentra cucullaria.-Dutchman's Breeches.-Petite Cote woods. (Holmes' corydalis cusullaria). May.

Corydalis glauca.-Pale Corydalis. - (Holmes). June. Mountain.

Nasturtium officinale, R.Br.-Water Cress:-Below bridge, near junction of cemeteries. July.

Nasturtium palustre, DC. - Marsh Cress. - (Holmes' sisymbrium palustre). June. Cummon.

Nasturtium armorica, Fries.-Hor'se Radish.-Creek near Cote des Neiges. June.

Dentaria diphylla, L.-Two-leaved Tooth wort.-Mountain, common. May and June. (Holmes).

Arabis levigata, DC.-Smooth Rock Cress.-Mountain, near Park Ranger's house. June. (Holmes' turritis levigata).

Erysimum cheiranthoides, L.-Wormseed Mustard.-Fletcher's Field. June.

Sisymbrium officinale, Scop.-Hedge Mustard-Common. June.

Brassica sinapistrum, Bois.-Charlock-Common. June. Brassica alba, L.-White Mustard.-(Holmes' sinapis alba). Common. June.

Brassica nigra, L.-Black Mustard.-(Holmes' sinapis nigra). Common. June.

Draba arabisans, Michx.-Whitlow Grass.-Outremont. June. 
Capsella bursa-pastoris, Mœnch.-Shepherd's Purse-Common. May. (Holmes' thlaspe bursa-pastoris).

Thlaspi arvense, L._Field Penny Cress.-(Holmes). Common. July.

Lepidium virginicum, L.-Wild Pepper Grass.-Common on streets. August. (Holmes).

Lepidium campestre, L.-Peppergrass.--Cote St. Louis. August.

Viola blanda, Willd.-Sweet White Violet.-Common. May. (Holmes).

Viola cucullata, Ait.-Common Blue Violet.-Common. May. (Holmes).

Viola pubescens, Ait.-Downy Yellow Violet.-Common. May. (Holmes).

Viola Canadensis, L.—Canada Violet.-Common. May. (Holmes).

Viola canina, L., var. Sylvestris, Regel.-Dog Violet.Hochelaga banks. May.

Helianthemum Canadense, Michx.-Frost Weed.-Petite Cote. June.

Hypericum perforatum, L.-Common St. John's Wort.Common. July. (Holmes).

Hypericum mutilum, L.-Small St. John's Wort.-Hochelaga banks. July. (Holmes'hypericum pa!viflorum).

Hypericum corymbosum, Muhl._Corymbed St. John's Wort.-Cote St. Paul. August.

Elodes virginica, Nutt.-Mar'sh St. John's Wort.-Bout de L'isle. August.

Saponaria officinalis, L.-Bouncing Bet.-Park Avenue. August.

Silene cucubalus, Wibel.-Bladder Campion.-Common. July.

Silene Pennsylvanica, Michx.-Wild Pink.-Field near Hochelaga bank. September. 
Silene noctitlora; L. - Night Flowering Catchfly.-Fletcher's Field. July.

Iychnis githag", Lam.-Corn Cockle.-Common. July.

Stellaria media, Smith.-Common Chickweed.-Common. July. (Holmes' alsine media).

Stellaria longifolia, Muhl. - Longleaved Stitchwort.-June. Cote St. Antoine. (Holmes' stellaria graminea).

Cerastium viscosum, L-Larger Mouse-ear Chickweed.June. Common. (Holmes).

Cerastium arvense, L. - Field Chickweed.-July. Common.

Cerastium nutans, Raf.-Nodding Chickweed.-Petite Cote. August.

Portulaca oleracea, L.-Common Purslano. - August. Common. (Holmes).

Claytonia Caroliniana, Michx. - Spring Beauty.-May. Common. (Holmes' C. Virginica).

Malva rotundifolia, L.-Round Leaved Mallow.-June. Common. (Holmes).

Tilia Americana, L.-Basswood.-May. Common.

Linum usitatissimum, L.-Common Flax.-Cote St. Louis and elsewhere. Strayed from cultivation. August.

Oxalis acetosella, L.-White Woodsorrel.-July. Hochelaga woods. (Holmes).

Oxalis stricta, L.-Yellow Woodsorrel._June. Common. (Holmes' O. Dillenii).

Impatiens fulva, Nutt.-Spotted Touch.me-not.-July. Common. (Holmes I. noli-me-tangere).

Impatiens pallida, Nutt.-Pale 'Touch-me-not.-August. Common. (Holmes I. biflora).

Zanthoxylum Americanum, Mill.-Northern Prickly Ash.April. Field near Petite Cote. (Holmes'Z. fraxineum).

Rhus typhina, L.-Staghorn Sumach.-Mt. Royal. July. (Holmes).

Rhus toxicodendron, L.-Poison Ivy.-June. Common. (Holmes). 
Vitis cordifolia, Michx.-Frost Grape.-June. Common. (Holmes' V. riparia).

Ampelopsis quinquefolia, Michx.-Virginia Creeper.Mountain and along fences 'on island. July. (Holmes' cissus hederacea).

Celastrus scandens, L.-Climbing Bitter-sweet.-June. Common on fences over the island. (Holmes).

Acer Pennsylvanicum, L._Striped Maple._May. North end of mountain.

Acer spicatum, Lam.-Mountain Maple.-May. Common. (Holmes).

Acer saccharinum, Wang.-Sugar Maple.-May. Lachine and elsewhere on island. (Holmes).

Acer dasycarpum, Ehrhart.-Silver Maple.-April. Common.

Acer rubrum, L.-Red Maple.-May. Common. (Holmes).

Trifolium pratınse, L.-Red Clover.-June. Common. (Holmes).

Trifolium repens, L.-White Clover.-June. Common. (Holmes).

Trifolium agrarium, L.-Hop Clover:-June. Hochelaga bank.

Trifolium procumbens, L.- Low Hop Clover. - July. Hochelaga bank.

Medicago lupulina, L._Black Medick.-June. Everywhere.

Melilotus officinalis, Willd.-Yellow Sweet Clover.-June. Everywhere.

Melilotus alba, Lam.-White Sweet Clover.-June. Common.

Robinia pseudacacia, L.-Common Locust.-June. St. Michel.

Desmodium acuminatum, DC.-Tick Trefoil._August. Mountain. (Holmes' Hedysarum acuminatum).

Desmodium Dillenii, Darling.-Tick Trefoil._August. Northern part of Mountain. 
Desmodium Canadense, DC.-Tick Trefoil.-August. Park near north end of mountain. (Holmes' Hedysarum Cana. dense).

Vicia sativa. L.-Common Vetch.-July. Common. (Holmes).

Vicia cracca, L.-Tufted Vetch.-June. Everywhere. (Holmes).

Amphicarpae monoica, Nutt.-Hog Peanut.-August. Overruns cemetery woods. (Holmes' glycine monoica).

Prunus Americana, Marshall.-Wild Plum.-Around mountain. May.

Prumus Pennsylvanica, L.-Wild Red Cherry.-Common. May. (Holmes).

Prunus Serotina, Ehrhart.-Wild Black Cherry.-Papineau Road and Petite Cote. June.

Prunus Virginiana, L.-Choke-cherry.-Very common. May. (Holmes' P. serotina).

Spirce salicifolia, L.-Common Meadow-sweet.-Common. August. (Holmes' S. latifolia).

Agrimonia eupatoria, L.-Common Agrimony.-July. Common. (Holmes).

Poterium Canadense, Benth and Hook.-Canadian Burnet. -Savanne, St. Michel. September. (Iolmes' sanguisorba Canadensis).

Geum album, Gmelin.-White Avens-.June. Hochelaga woods. (Holmes).

Geum strictum, Ait.-Yellow Avens.-June. Common. (Holmes).

Greum rivale, I - Purple Avens. - July. Common. (Holmes).

Potentilla Norvegica, L.-Norway Cinque-foil.-July. Common. (Holmes).

Potentilla Canadensis, L. - Canada Cinque foil. - June. Hochelaga bank. (Holmes' P. simplex).

Potentilla anserina, L.-Silver Weed.-June. Common. (Holmes). 
Potentilla fruticosa, L.-Shrubby Cinque-foil._Savanne, St. Michel. September. (Holmes).

Fragaria Virginiana, Ehrhart.-Common Strawberry.May. Common. (Holmes).

Fragaria vesca, L.-Sharp Pointed Strawberry.-June. Common in woods.

Rubus odoratus, L.-l'urple Flowering Raspberry.-Mountain and elsewhere. June. (Holmes).

Rubus triflorus, Richardson.-Dwarf Raspberry.-Hochelaga banks and elsewhere. May. (Holmes).

Rubus strigosus, Michx.-Wild Red Raspberry.-June. All over the island. (Holmes).

Rubus occidentalis, L.-Black Raspberry.-East side of mountain. June. (Holmes).

Rubus villosus, Ait.-Higb Blackberry.-North end of mountain. June. (Holmes).

Rubus hispidus, L.-Running Swamp Blackberry.-Swamp between cemeteries. June.

Rosa blanda, Ait._Early Wild Rose.-East end of mountain. June.

Rosa Carolina, L.-Swamp Rose.-Papineau woods. July. (Holmes).

Rosa rubiginosa, L.-Sweet Brier.-Mountain, Outremont and elsewhere. June. (Holmes).

Cratagus coccinea, L.-Scarlet Fruited Thorn.-Mountain. May. (Holmes).

Cratagus tomentosa, L.-Black or Pear Thorn.-North end of mountain and elsewhere. June. (Holmes).

Cratagus crusgalli, L.-Cockspur Thorn.-North ond of mountain. June. (Holmes).

Pyrus arbutifolia, L.-Chokeberry.-Cote St. Paul. June. (Holmes' aronia melanocarpa).

Pyrus americana, D. C.-American Mountain Ash.-June. (Holmes' sorbus Amoricana). 
Amelanchier Canadensis, var. rotundifolia, Torr and Gray. -shadbush.-May. Mountain. (Holmes' aronia ovalis). Amelanchier Canadensis, var. Botryapium, Torr and Gray. -Tuneberry.-May. Inchelaga banks. (Holmes' aronia botryapium).

Ribes cynosbati, L.-Wild Gooseberry.-Between cemeteries. May. (Holmes' ribes triflorum).

Ribes hirtellum, Michx.-Small Wild Gooseberry.-June. St. Michel.

Ribes lacustre, Poir.-Swamp Gooseberry.-Hochelaga banks. June. (Holmes).

Ribes floridum, L.-Wild Black Currant. May. Between cemeteries. (Holmes).

Ribes rubrum, L.-Wild Red Currant.-May. Hochelaga banks. (Holmes).

Parnassia Caroliniana, Michx._Grass of Parnassus.-Deptember. Savanne, St. Michel.

Saxifraga Virginiensis, Michx.-Early Saxifrage.-May. Mountain. (Holmes' S. nivalis).

Mitella diphylla, I.-Two-leaved Mitre-wort.-Base of mountain. May. (Holmes).

Mitella nuda, L.-Naked Stalked Milella.-Hochelaga woods. June. (Holmes M. cordifolia).

Tiarella cordifolia, L.-False Mitre-wort.-May. Base of mountain. (Holmes).

Penthorum sedoides, Gronov.-Ditch Stonecrop.-Cote St. Paul. July. (Holmes).

Circeea lutetiana, L.-Enchanter's Nightshade.-Hochelaga woods. July. (IIolmes).

Circcea Alpina, T.-Small Enchanter's Nightshade.Mountain. July. (Holmes).

Epilsbium angustifolium, L._Great Willow Herb.-July. ITochelaga woods and elsewnere. (Holmes).

Epilobium palustre, T., var. lineare.-Epilobo.-A Agust. (Holmes). 
Epilobium molle, Torr.-Epilobe-_August. Cote St. Paul. Epilobium coloratum, Muhl. - Epilobe Swamp. - St. Michel. (Holmes' E. tetragonum).

Enothera biennis, L.-Common Evening Primr'sse.August. St. Michel and elsewhere. (Holmes).

Enothera pumila, L._Small Evening Primrose.-Hochelaga bank. June. (Holmes' (E. pusilla).

Sicyos angulatus, L.-Star Cucumber.-Fletcher's Field. September. (IIolmes).

Sanicula Marilandica, L.-Snakeroot.-June. Mountain. (Holmes).

Daucus carota, L.-Common Carrot.-June. Road-sides.

Heracleum lanatum, Michx.-Cow Parsnip.—Cote St. Paul and elsewher'e. June. (Holmes).

Pastinaca sativa, L.-Common Parsnip.-June. Roadsides. (Holmes).

Conioselinum Canadense, Torr. and Gray.-Hemlock Parsley.-Back River and elsewhere.

Cicuta maculata, L.-Spotted Cowbane.-Cote St. Paul. July. (Holmes).

Sium lineare, Michx. - Water Parsnip.-August. Pointeaux-Trembles. (Holmes).

C'arum carui, L. - Caraway.-June. Roadsides, in many places.

Osmorrhiza longistylis, DC.-Smoother Sweet Cicely.June. Mountain side. (Holmes' Myrrhis longistylis).

Osmorrhiza brevistylis, DC._Hairy Sweet Cicely.-June. Mountain side. (Holmes' chærophyllum Claytoni).

Aralia racemosa, L.-Spikenard.- North end of mountain. July. (Holmes).

Aralia nudicaulis, L._Wild Sarsapar:Ila._Jụne. Moun. tain sides and elsewhere. (Holmes).

Aralia quinquefolia, Decaisne. - Ginseng. - Hochelaga woods. May. (Holmes' panax quinquefolia). 
Aralia trifolia, Decaisne.-Dwarf Ginseng.-Hochelaga woods and elsewhere. (Holmes).

Corms Canadensis, L.-Bunchberry.-Papinean woods. . Iune. (Holmes).

Cornus circinnata, L'Her.--Round-leaved Dogwood.Outremont road. Iune. (Holmes).

Cornus stolonifera, Michx.-Redosier Dogwood.-Tuly. Common. (Holmes' C. alba).

Cimmea Borcalis, Gronov.-Twin-flower. - Hochelaga woods and Cote Michel. July. (Holınes).

Lonicera parviflora, Lam.-Sraall Honeysuckle.-June. Mountain. (Holmes).

Lonicera ciliata, Muhl.-Fly Honeysuckle.-North end of mountain. May. (Holmes' Xylosteon ciliatum).

Lonicera oblongifolia, Mubl._-Swamp Fly-Honeysuckle.Buchanan's woods, St. Michel. August. (Holmes).

Diervilla trifida, Mœnch.-Bush Honeysuckle.-July. Mountain. (Holmes).

Sambucus Canadensis, L.-Common Elder.-Mountain sides and elsewhere. Tune. (Holmes).

Sambucus racemosa, L.-Redberried Elder.-May. Mountain sides and elsewhere. (Holmes' S. Pubescens).

Viburnum opulus, I.-Cranberry Tree.-June. Back River road. (Holmes' V. Oxycnccus).

Viburnum Lentago, L.-Sheepberry.-Cote St Paul. June. (Holmes).

Viburnum acerifolium, L.-Maple-leáved Arrowwood.June. Monté between St. Michel and Back River. (Holmes).

Lappa officinalis, All., val. Major, Gray.-Burdock.-June. Everywhere. (Holmes' aretium lappa).

Cirsium lanceolatum, Scop.-Common Thistle.-August. Everywhere.

Cirsium discolor, Spreng.-Tall Thistle.-August. St. Michel. (Holmes' Cuicus altissimus). 
(Yirsium muticum, Michx.-Swamp Thistle-August. St. Michel. (Holmes' cnicus muticus).

Cirsium arvense, Scrp.-Canada Thistle.-August. Everywhere. (Holmes' cnicus horridulus).

Xanthium strumarium, L., var. Echinatum, Gray.-Common Cocklebur. August. Common. (Holmes).

Ambrosia artemisicefolia, L.-Hogweed.-August. Everywhere. (Holmes).

Ambrosia trifida, L.-Great Ragweed.-August. Common. (Holmes).

Tanacetum vulgare, L --Common Tansy.--August. Roadsile in many places.

Artemisia vulgaris, L.-Common Mugwort. - August. Everywhere. (Holmes).

Gnaphalium decurrens, Ives.-Everlasting.-August. Common.

Gnaphalium polycephalum, Michx.-Common Eveslasting. -August. Common.

Grnaphalium uliginosum, L.-Low Cudweed.-August. Common. (Holmes).

Antennaria plantaginifolia, Hook.-Plantain-leaved Everlasting.-May. Everywhere. (Holmes' Gnaphalium plantaginifolium.

Eupatorium purpureum, T.-Joe Pye Weed.-August. Mountain Park, Cote St. Paul. (IIolmes' E. verticillatum).

Eupatorium perfoliatum, I. - Boneset.-August. Mountain Park, Cote St. Antoine. (ILolmes).

Eupatorium ageratoides, I.-White Snakeroot.-August. Mountain Park, Cote St. Michel. (Irolmes).

Senecio vulgaris, I.-Common Groundsel,-On all streets. Tuly. (Holmes).

Senecio aureus, I.-Golden Ragwort.-August. St. Michel. Solidago squarrosa, Muhl._Golden Rod._East slope of Mountain. August.

Solidago bicolor, L., var., Concolor.-Golden Rod.-Míountain base. August. (Holmes). 
Solidago latifolia, L.-Golden Rod.-Mountain base. August. (Holmes).

Solidago Oeesia, L., var. axillaris, Gray.-Golden Rod.Mountain base. August. (Holmes' S. livida).

Solidago Canadensis, L.-Golden Rod.-August. Common. (Holmes).

Solidago lanceolata, L.-Golden Rod.-August. Mountain base. (Holmes).

Aster macrophyllus, I. - Starwort. - Mountain sides. August. (Holmes).

Aster azureus, Lindl.-Aster.-Cote St. Paul. August. Aster cordifolius, T. - Aster. - August. Everywhere. (Holmes).

Aster sagittifolius, Willd.-Aster.-September. Very common. (Holmes).

Aster kevis, L.--Aster.-August. Mountain.

Aster puniceus, L.-Aster. - August. Cote St. Paul. (Holmes).

Aster multiflorus, Ait.-Aster.-August. Cote St. Paul.

Aster tenuifolius, I.-Aster.-August. Mountain base.

Aster acuminatus, Michx. - Starwort. - Park woods. August.

Erigeron Canadense, I.-Horseweed.-August. Papineau Road. (Holmes).

Erigeron bellidifolium, Muhl.--Robin's plantain.-July. Mountain.

Erigeron Philadelphicum, L.-Common Fleabane.-June. South Mountain foot. (Holmes' E. purpureum).

Erigeron strigosum, Muhl. - Daisy Fleabane. - June. Pointe-aux-Trembles. (Holmes).

Erigeron annuum.-Larger Daisy Fleabane.-Cote des Neiges. August. (Holmes' E. heterophyllum).

Leucanthemum vulgare, Lam. - Oxeye Daisy. - June. Everywhere. (Holmes' chrysanthemum). 
Anthemis cotula, DC.-Maywced.-May. Ererywhere. (Holmes).

Rudbeckia hirta, L.-Coneflower.-Petite Cote. July.

Helianthus divaricatus, L.-Wild Sunflower.-August. Cemeteries and Park.

Helianthus tuberosus, L.-Jerusalem Artichoke. -August. Prince Arthur street.

Bidens frondosa, L. - Common Beggar Ticks.-July. Fletcher's Field. (Holme's B. pilosa).

Bidens Connata, Mubl._Swamp Beggar Ticks.-August. Cote St. Paul. (Holmes).

Bidens cernua, L.-Smaller Bur-marigold._August. Long Pointe. (Holmes).

Achillea millefolium, L._Milfoil._June. Everywhere. (Holmes).

Cichorium Intybus, L.-Cichory.-July. Everywhere. (Holmes).

Hieracium Canadensis, Michx.-Canada Hawkweed.August. Cemeteries. (Holmes' H. kalmii).

Hieracium scabrum, Michx.-Rough Hawkweed.-August. Papineau Road and elsewhere. (Holmes' H. Mariunum). Nabalus albus, Hock.-White Lettuce.-August. Common. (IIolmes' prenanthes alba).

Nabalus altissimus, Hook.-Tall White Lettuce.-August. North end of mountain. (Holmes' prenanthes cordata).

Nabalus racemosus, Ilook.-Rattlesnake Root.-August. Mountain Park and Cote St. Paul. Common. (Holmes' Prenanthes racemosa).

Taraxacum dens-leonis, Desf.-Common Dandelion.-May. Everywhere. (Holmes' leontodon taraxacum).

Lactuca Canadensis, L.-Wild Lettuce.-North end of mountain. August. (Holmes' lactuca elongata).

Lactuca integrifolia, L.-Wild Lettuce.-August. Cemetery swamp. (Holmes' L. elongata).

Mulgedium leucophceum, DC.-False or Blue Lettuce.- 
North end of mountain. August. (Holmes' sonchus lencophrus).

Sonchus oleraceus. L.-Common Sow-thistle.-July. Common. (Holmes).

Sonchus Asuer, Till.-Spring-leaved Sonchus. August. Fletcher's Field and Park.

Sonchus arvensis, 1._Field Sow-thistle.-Cote St. Antoine and St. Michel. August. (Holmes).

Tragopon pratensis, L.-Yellow Goat's Beard.-July. Longue Pointe.

Lobelia cardinalis, L.-Cardinal Flower.-August. St. Michel. (Holmes).

Lobelia inflata, L.-Indian 'Tobacco.-August. Plateau, north end of mountain. Common. (Holmes).

Campanula Americana, L.-Tall Bell-flower.-Petite Cote and elsewhere on roadsides. August.

Vaccinium corymbosum, L.-Swamp Blueberry. - Hochelaga banks. June. (IIolmes).

Pyrola rotundifolia, L.-Wintergreen.-Mountain. July. (Holmes.)

Pyrola elliptica, Nutt.-Shinleaf. - Hochelaga woods. July. (Holmes).

Pyrola secunda, var. pumila, L.-Wintergreen.-Hochelaga woods. July. (Holmes).

Chimaphila umbellata: Nutt.-Prince's Pine.-August. Mountain, west side near Cemetery and Buchanan's woods, St. Nichel, among pines. (Holmes' pyrola umbellata).

Plantago major, L.-Common Plantain.-August. Common. (IIolmes).

Plantago lanceolata, L.-Rib-grass.-August. Common.

Trientalis Americana, Pursh.-Star-flower.--June. Hochelagra woods. (Holmes).

Lysimachia Thyrsiflora, L.-Tufted Toosestrife.- June. Pack River. (Holmes' I. capitata).

Lysimachia stricta, Ait.-Loosestrife.-June. Cote St. Paul. (IIolmes' L. racemosa). 
Lysimachia longifolia, Watt.-Loosestrife.-August. St. Michel.

Verbascum thapsus, L.-Common Mullein.-July. Common. (Holmes).

Veronica anagallis, L. - Water Speedwell. - Lachine. July. (Holmes).

Veronica scutellata, L.-Mar'sh Speedwell.-August. St. Michel. (Holmes).

Veronica peregrina, L.-Neckweed.-May. Common. (IIolmes).

Veronica serpyllifolia, I.--Thyme-leaved Speed well.--May. Hochelaga banks. (Holmes, at Berthier).

Lintria vulgaris, Mill.-Toad Flax.-July, Common on str'eets.

Scrophularia nodosa, L. - Figwort. - June. Common. (Holmes' S. Marilandica).

Chelone glabra, L.-Turtle Head.-August. St. Michel and batween cometeries.

Mimulus ringens, L. - Monkey Flower. - Hochelaga. July. (Holmes).

Gerardia tenuifolia, Valıl.—Silender Gerardia.-September. Savanne. St. Michel.

Pedicularis Canadensis, L. - Wood Betony. - May. Mountain base. (Holmes).

Verbena hastata, L.-Blue Vervain.-Hocheliuga banks. June. (Holmes).

Verbena urticifolia, L.-Nettleleaved Vervain.-North base of mountain. August. (Holmes).

Phryma leptostachya, L.-Lopseed.-Savanne. St. Michel and mountain. August. (Holmes).

Teucrium Canadense, L.-American Germander.-August. St. Michel. (Holmes, at Boucherville).

Mentha viridis, L.-Spearmint.-Petite Cote. August. (Holmes' M. tenuis).

Mentha piperita, L.-Peppermint.-Point St. Charles. August. 
Mentha Canadensis, L.-Wild Mint.-August. Common. (Holmes' M. borealis).

Lycopus Vrginicus, L.-Bugle-weed.-Hochelaga woods. August. (Holmes).

Lycopus Europcus, L., var. Sinuatus, Gray. - Water Horehound.-August. Common. (Holmes).

Nepeta cataria, L.-Catnip.-July. Common. (Holmes).

Nepeta glechoma, Benth.-Ground Ivy.-Fletcher's Field and elsowhere, near houses.

Lophanthus nepetoides, Benth.--Giant Hyssop.-August. Mountain, near Cote des Neiges toll bar. (Holmes' Hyssopus petoides).

Brunella vulgaris, L.--Common Heal-All.-July. Everywhere. (Holmes' prunella vulgaris).

Scutellaria galericulata, L. - Skullcap. - St. Michel. August. (Holmes).

Scutellaria lateriflora, L.-Mad-dog Skullcap.-August. St. Michel. (Holmes).

Galeopsis tetrahit, L.-Common Hemp-nettle.-August, Common. (IIolmes).

Stachys palustris, L., var. Aspera, Gray.-Hedgo-nettle.Fletchor's Field. (Hulmes, at Boucherville Island).

Leonurus cardiaca, L.-Common Motherwort.-August. Papineau cornmon and elsewhere. (Holmes).

Lycopsis arvensis, L. - Bugloss. - June. Common. (Holmes).

Symphytum officinale, L.-Common Comfrey.-East side of mountain, near Cote des Neiges Road. June.

Echinospernum lappula, L. Lehm.-Stickseed.-June. Common. (Holmes' Myosotis Lappula).

Cynoglossum officinale, L.-Common Hound's Tongue.June. Common. (Holmes).

Lithospermum hirtum, Lehm. - Hairy Puccoon.-May. Common.

Lithospermum officinale, L.-Common Gromwell.-May. Common. (Holmes). 
Myosotis palustris, Withering, var. laxa, Gray.-Forgetme-not.-Pointe-aux-Trembles. June.

Myosotis arvensis, Hoffm.-Field Myosotis.-Cemetery Swamp. August.

Hydrophyllum Virginicum, L.-Waterleaf.-June. Base of mountain and elsewhere. (Holmes).

Calystegia sepium, R. Br.-Hedge Bindweed.-St. Michel Road. August. (Holmes' convolvulus sepium).

Calystegia spithancea, Pursh.-Bracted Bindweed.-Back River. June. (Holmes' convolvolus stans, at Three Rivers.)

Convolvulus arvensis, L.-Bindweed.-July. Fletcher's Field.

Hyoscyamus niger, L.-Black Henbane.-St. Famille street, Fletcher's Field and elsewhere. (Holmes).

Datura stramonium, T.-Common Thorn Apple.-St. Lawrence suburbs. August. (Holmes).

Gentiana Andrewsii, Griseb.-Closed Gentian.-St. Michel. August.

Apocynum androsamifolium, L.-Dogbane.-June. Mountain. (Holmes).

Asclepias Cornuti, Decaisne.-Common Milkweed.-July. Common. (Holmes' A. Syriaca).

Fraxinus Americana, L.-White Ash.-Between Petite Cote and St. Michel, along fence. June. (Holmes' F. Epiptera).

Fraxinus pubescens, Lam.-Red Ash.-Same locality. June.

Fraxinus sambucifolia, Lam.-Black Ash.-St. Michel woods. June. (Holmes).

Chenopodium album, L.-Tamb's Quarters.-July. Everywhere. (Holmes).

Chenopodium murale, L.-Pigweed._August. Upper St. Urbain street.

Chenopodium hybridum, I.-Maple-leaved Goosefoot.West side of mountain. August. (Holmes). 
Blitum capitatum, I. - Strawberry Blite.-Fletcher's Field. August. (Holmes).

Atriplex patula, L.-Orache.-July. Everywhere on streets. (Holmes).

Amarantus, retrotlexus, L.-Pigweed.-August. Everywhere. (Holmes).

Polygonum aviculare, T._(ioose-grass.-August. Everywhere. (Holmes)

Polygonum incarnatum, Ell.-Knotweed.-August. Cote St. Paul.

Polygonum Pennsylvanicum, L -Knotweed.-August. St. Urbain street. (Holmes).

Polygonum Persicaria, L._Lady's Thumb._July. Everywhere. (Holmes).

Polygonum amphibium, var. aquaticum, L.-Water Persicarria.-St. Michel. August. (Holmes).

Polygonum hydropiperoides, Michx.-Mild Water Pepper. -August. Cote St. Paul. (Holmes).

Polygonum acre, H. B. K.-Water Smartweed.-Petite Cote. August.

Polygonum hydropiper, L.-Common Smartweed.--August. Common.

Polygonum sagittatum, L--Arrow-leaved Tear Thumb.Mountain. August. (Holmes).

Polygonum convolvulus, I.-Black Bindweed.-Grain fields. August. (Holmes).

Polygonum dumetorum, T., var'. Scandens, Gray.-Climbing False Buckwheat.-Cote St. Paul. (Holmes' P. Scandens).

Rumex orbiculatus, Gray._Great Water Dock._-St. Michel, Savanne. Augrust.

Tumex crispus, I. - Curled Dock. - July. Common. (Holmes).

Rumer, obtusifolius, L.-Bitter Dack.-Cote des Neiges. August. (Holmes).

Rumex acetosella, L.--Sheep Sorrel.-May. Everywhere. (IIolmes). 
Fagopyrum esculentum, Mœnch.-Buckwheat.-Fletcher's Field and elsewhere. August.

Dirca palustris, L.-Leatherwood.-Petite Cote. April. (IIolmes).

Euphorbia hypericifolia, L.--Spur'ge.-August. Fletcher's Feld and elsewhere.

Euphorbia humistrata, Engelm. - Spurge. - August. Fletcher's Ficld.

Euphorbia obtusata, Pursh. - Spurge.-St. Tawrence suburbs. August.

Euphorbia platyphylla, T. - Spurge. - St. Lawrence suburbs. August.

Euphorbia Helioscopia, T._Sunspurge.—Jnly. Everywhere. (Holmes).

Euphorbia peplus, I.-Spurge.-Angnst. Fletcher's Field and mountain base.

Euphorbia cyparissias, L.-St. Famille street and elsewhere. July.

Acalypha Virginica, Is-Three Seeded Nercury.-St. Famille strect and elsewhere, very common. August. (Holmes' A. Caroliniana).

Ulnus fulva, Michx.-Slippery Elm.-April. Common. (IIolmes).

Ulmus Americana, L.-White Elm.-April. Common. (Holmes).

Ulmus racemosa, Thomas.-Corky White Elm.-May. Along roadsides.

Urtica gracilis, Ait.-Nettle.-August. Along fences. (Holmes' U. procera).

Laportea Canadensis, Gandichaud. - Wood Nettle. August. Back River. (Holmes' Urtica divaricata).

Cannabis sativa, L.-ITemp.-July. Common. Along roadsides. (Holmes).

Juglans cinerea, L.-Butternut.-Mountain sides and Petite Cote. (Holmes). 
Carya amara, Nutt.-Swamp Hickory.-Petite Cote and mountain. May. (Holmes).

Caryc ulba, Nutt._Shellbark Hickory.-West side of mountain. May. (Holmes).

Quercus rubra, T.-Led Oak.-May. Mount Royal. (Holmes).

Quereus macrocarpa, rill Olivaformis, Gray.-Bur Oak.May. St. Tamrent and elsewhere. (Holmes).

Fagus ferruginea, Ait.-American Beech.-May. West side of Mount Royal. (Holmes).

Corylus rostrata, Ait.-Beaked Hazel Nut.-June. Base of mountain. (Holmes' C. anellana).

Ostrya Virginica, Willd.-Iron Wood.-May. Mountain. (Holmes).

Betula lenta, I._Black Birch.-May. Hochelaga woods.

Betula pamyracea, Ait.-Canoe Birch.-May. Mountain. (Holmes).

Betula populifolia, Ait.-Gray Birch.-Petite Cote. May. (Holmes).

Alnus incana, Wiild.-Speckled Alder.-April. Mountain foot. Common. (Holmes' A. serrulata).

Alnus viridis, DC._Green Alder.-Between mountains. May. (INolmes' A. undulata).

Salix humilis, Marshall.-Prairie Willow.-Hochulaga bank. May.

Salix discolor, Muhl._Glaucous Willow.-North base of mountain.

Salix petiolaris, Smith.-Petoiled Willow._Savanne, St. Michel. August.

Salix livida, Wahl., var. occidentalis, Gray.-Livid Willow.-All over the island. May.

Populus tremuloides, Michx.-American Aspen.-May. All over the island. (Holmes).

Populus balsamifera, L.-Balsam Poplar.-May. Common. 
Populus monilifera, Ait.-Cottonwood.-Upper St. Famille street. May. (Holmes' P. angulata).

Populus monilifera, Ait., var. Candicans, Gray.-Balm of Gilead.-May. St. Famille street.

Populus alba, L.-White Poplar.-Cote St. Antoine and clsewhere. May.

Pinus strobus, L.-White Pine--May. Savanne, St. Michel. (Holmes).

Pinus Banksiana, Lambert. - Scrub Pine.- Mountain. May.

Abies balsamea, Miller.-Balsam Fir.-Hochelaga woods. May. (Holmes' pinus balsamea).

Picea nigra, Link.-Black Spruce.--Petite Cote. May.

Picea alba, Link.-White Spruce.-St. Michel. May.

Tsuja Canadensis, Carr--Hemlock.-Hochelaga woods. May.

Larix Americana, Michx. - Tamarack. - Petite Cote Swamp. May.

Thuja occidentalis, I._Cedar.-May. In all swamps on island.

Juniperus communis, L. - Common Juniper:-Between mountains. August.

Taxus Canadensis, L., var. Canadensis, Gray.-Ground Hemlock.-Mountain. May. (Holmes).

Arisama triphyllum, Torr.-Indian Turnip.-Juno. Mountain swamp. (Holmes' Arum triphyllum).

Calla palustris, L.-Marsh Calla.-Petite Cote Sivamp. June.

Typha latifolia, I.-Common Cat Tail.-Pointe-auxTrembles and elsewhere. July. (Holmes).

Typha angustifolia, L.-Narrow-leaved Cat Tail.-Pointeaux-Trembles. June.

Sparganium simplex, Hudson, var. Angustifolium, Gray.Bur Reed.-June. Back River. 
Alisma plantago, L., var. Americanum, Gray.-Water Plantain.-July. Cote St. Paul. (ITolmes).

Sagittaria variabilis, Engelm.-Arrowhead.-July. Common. (Holmos' S. sayittifolia).

Habenaria tridentata, Hook.-Rein Orehis.-Woods noar cemetery giate. June.

Habenaria orbiculata, Torr. - Rein Orchis. - Between cemeteries. July.

Spiranthes Romanzoviana, Chamisso. - Ladies' 'Tresses.Saranne, St. Wichel. July.

Cypripedium pubescens, Willd. - Large Yellow Lady's slipper.-West side of mountain. June. (Holmes).

Cypripedium acaule, Ait. -Stemless Laly's Slipper.-June. Hochelagia woods. (IIolmes' C. arietinum).

Iris versicolor, L._Larger Blue Flag.-June. Common.

Sisyrin.hium anceps, Cav.-Blue eyed Grass.-June. Common. (Holmes).

Smilax herbacen, L.-Carrion Flower.-St. Laurent. June.

Trillium granditorum, Salisb.-Largo White Trillium.May. Very common. (IIolmes).

Trillium erectum, L.-Purple Trillium.-Mountain, Hocholaya woods and elsewhere. (Holmes).

T'rillium erythrocarpum, Michx._Pointed Trillium._May. Hochelaga woods. (Holmes).

Medeola Viryinica, L.-Indian Cucumber Root.-June. Hochelaga woods. (ILolmes).

Uvularia grandiflora, Smith.-Bellwort.-Papineau Road and mountain. Mily. (Holmes).

Uvularia sessilifolia, L._Wood Daffodil._- Vay. North end of mountain. (Holmes).

Clintonia borealis, Raf. - Clintonia. - Junc. Mountain marsh and Hochelaga woods. (Holmes' convallaria bol'calis).

Streptopus roseus, Michx.-Twisted Stalk-May. Moun- 
tain and Hochelaga woods. (Holmes' convallaria polygonatum).

Smilacina racemosa, Desf.-False Spikenard. North end of mountain. August. (Holmes' convallaria racemosa).

Smilacina stellata, Desf.-False Solomon's Scal.-Lachine and elsewhere. (Holmes' convallaria stellata).

Smilacina bifolia, Ker., var. Canadensis, Gray.-False Solomon's Seal.--June. Mountain and Hochelaga woods. (Holmes' convallaria bifolia).

Polygonatum biflorum, Ell.-Smaller Solomon's Seal.May. West side of mountain. (Holmes' convallaria angustifolia).

Erythronium Americanum, Smith. - Yellow Adder's Tongue.-May. Mountain and elsewhere. (Holmes' E. dens-canis).

Polypodium vulgare, L.-Polypody.-North end of mountain. June. (Holmes).

Adiantum pedatum, L.-Maidenhair.-June. Mountain. (Holmes).

Pteris aquilina, L. - Brake. - August. Common. (Holmes).

A.splenium filix famina, Bernh.-Spleenwort.-Mountain base.-June. (Holmes' A. angustifolium).

Aspidium thelypteris, Swartz.-_Shield Fern.-Mountain base. (Holmes' athyrium thelypteris).

Aspidium Noveboracense, Swairtz.--Wood Fern.-Mountain foot. June.

Aspidium marginale, Swartz. — Shield Fern. - Mountain base. July. (Holmes).

Onoclea sensibilis, L.-Sensitive Fern.-August. Hochelaga woods. (Holmes).

Osmunda regalis, L. - Flowering Fern. - Hochelaga woods. (Holmes).

Osmunda Claytoniana, L.-Osmunda.-Angust.-Hochelaga woods. (Holmes' O. interrupta). 
Osmunda Cinnamomea, L.-Cinnamon Fern-Dugust. Hochelaga woods. (Holmes).

Botrychium Virginicum, Swartz.-Moonwort.-July. North end of mountain. (Holmes' B. Gracile).

Equisetum limosum, L.-Horse Tail-_May. Hochelaga. (Holmes).

Equisetum arvense, L.-Common Horse Tail.-May. liverywhere. (Holmes).

Equisetum hyemale, L.-Scouring Rush.-June. North base of mountain.

Equisetum variegatum, Schleicher.-Horse Tail.-June. Cote St. Paul.

Equisetum scirpoides, Michx.-Horse Tail.-Mountain base. July.

Lycopodium dendroideum, Michx.-Ground Pine.-August. Hochelaga banks. (Holmes).

Lycopodium clavatum, I.-Club Moss.-August. Hochelaga woods. (Holmes).

Lycopodium lucidulum, Michx._Club Moss._Savanne, St. Michel. August. (Holmes). 





